\title{
Evidence for a significant contribution of interactions between oriented line segments in the Tolansky version of the Poggendorff illusion
}

\author{
PETER WENDEROTH, TONY O'CONNOR, and MICHAEL JOHNSON \\ University of Sydney, Sydney, New South Wales, Australia
}

\begin{abstract}
Interactions between line segments, including angle distortion, commonly are proposed as partial determinants of the Poggendorff illusion. Day and Kasperczyk (1985) found that replacing all transverse line segments with dots had no effect on alignment errors, thus rendering untenable explanations couched solely or largely in terms of line interactions. The illusions they obtained, using Tolansky variants of the basic illusion, were attributed to judgments that compromised between vertical and oblique bisection. In the present experiment, subjects were instructed to avoid such a compromise: Preliminary experiments had suggested that compromise judgments were more likely to affect displays with dot pointers than those with line pointers. It was found that the addition of parallel inducing lines significantly increased illusions, but only when transverse pointers were line segments rather than dots. This was consistent with previous research, which has shown that line interactions are a significant component of Poggendorff effects.
\end{abstract}

The conventional form of the Poggendorff illusion and the Tolansky variant of it are shown in Figures 1a and $1 \mathrm{~b}$, respectively. In the former, the upper and lower segments of the vertical test line that passes behind the inducing parallels are collinear, but appear not to be so; in the latter, the upper vertical test line appears not to bisect the horizontal distance between the two lower vertical lines, yet it does so in fact.

Although these illusions, like many others, are larger when the test line segments are oblique rather than vertical, Day and Kasperczyk (1985) cleverly used variants of Figures $1 \mathrm{a}$ and $\mathrm{lb}$ in an attempt to demonstrate that the test line segments play no role in the illusions, because equivalent effects occurred when the test lines were replaced with dots. Specifically, they claimed to have shown that equal errors occur when observers attempt vertically to bisect the horizontal distance between two vertical lines with an upper line (Figure 1b) or an upper dot (Figure 1c); or when the lower targets are dots with an upper vertical pointer (Figure 1d); or even when the upper pointer and the lower targets are all dot stimuli (Figure 1e). In every case, the observers judged the upper pointer to be too high and to the left when it was physically above the horizontal midpoint between the lower targets; and so they set the pointer too far down and to the right (a positive error).

This research was supported by a University of Sydney Special Projects Grant in 1985 and by the Australian Research Grants Scheme (Grant A28515620) in 1986, both of which provided funds for research assistance by the second author.

The authors' mailing address is: Department of Psychology, University of Sydney, N.S.W., Australia 2006.
The finding that identical-magnitude illusions occurred for Figures 1b, lc, 1d, and 1e led Day and Kasperczyk to conclude that theories of the Poggendorff illusion that seek to explain it in terms of angular effects or line displacements are untenable (see Day \& Kasperczyk, 1985, for a comprehensive review of these theories). In addition, they proposed an alternative explanation. The upper dot in Figure le can be set to bisect the space between the two lower dots in two ways. First, it can do so in the manner required of the observers, such that a vertical plumb line dropped from it would bisect the horizontal distance between the lower dots (vertical bisection). Second, the upper dot could be set so that a perpendicular dropped from its position on the upper line to the oblique line joining the two lower dots bisects that line (oblique bisection). Day and Kasperczyk suggested that, for all four stimulus displays, observers chose a compromise between these two possibilities, resulting in the upper pointer's being set too low and too far to the right.

Several aspects of the Day and Kasperczyk results led us to conduct our own experiments using similar figures. First, although it is plausible to suppose that the tendency to confuse vertical and oblique bisection could occur with the all-dot display (Figure 1e), this seems less likely to occur with the other figures in which lines at least partially define the direction of bisection. Consequently, average errors for the all-dot display might have equaled those in the other conditions because more subjects in the alldot condition mistakenly attempted the oblique bisection task. Credence is given to this suggestion by the fact that in Day and Kasperczyk's first experiment, using repeated measures, the standard deviation for the three-dot condition (2.06) was more than three times larger than that for 
a

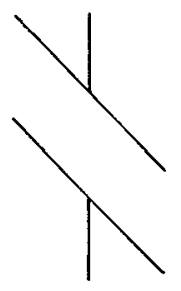

c

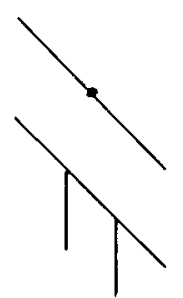

Figure 1. Variations of the Poggendorff illusion. (a) The conventional form with single transversal. (b) The Tolansky variant. (c, d, e) Tolansky variants in which some or all line transversals are replaced with dots.

the three-line condition (0.67). In their second experiment, using independent groups, the standard errors were comparable but the three-dot mean was smaller, albeit not significantly so.

Second, although our data subsequently proved us wrong, casual inspection of the various Figure 1 stimuli without oblique parallels seemed to indicate that the illusion was much stronger in the parallel-less three-dot version than in the parallel-less three-line figure. If this were so, one might conclude that the with-parallels effects were all equivalent in magnitude not because lines were irrelevant but because the addition of parallels increased line illusions more than dot illusions. In other words, angular or line displacement effects could be a key component of the Poggendorff illusion, even if the illusions induced in figures with parallels were all equal.

Third, when we began experiments on these effects, we noted that when the upper pointer was a dot, some subjects did attempt oblique rather than vertical bisection. This did not occur with upper line pointers. Consequently, we began again, explained the distinction between vertical and oblique bisection to all subjects, and instructed them to attempt only vertical bisection and not to confuse this with oblique bisection.

In brief, the purpose of this study was (1) to ascertain whether equivalent effects occur with Figures $1 \mathrm{~b}$ to $1 \mathrm{e}$ when subjects explicitly are instructed to avoid oblique bisection, and (2) to discover whether smaller effects occur with some figures (specifically figures with vertical line segments) when the oblique parallels are deleted. We chose also to use inverted figures, with a fixed pointer below and adjustable targets above. This was done because some Poggendorff illusions are larger with inverted figures (Tong \& Weintraub, 1974) than with the upright figures.

\section{METHOD}

\begin{abstract}
Apparatus
Stimulus figures were presented on the flat face of a Tektronix 608 display monitor (P4 phosphor) stationed $114 \mathrm{~cm}$ from the observer's padded head- and chinrest, so that $2 \mathrm{~cm}$ on the screen subtended $1^{\circ}$ of visual angle. The subject had control of three microswitches, the outer pair of which could be used to move the adjustable portion of each display left or right along an oblique track. The display screen was interfaced with a Hewlett-Packard 1350A graphics translator and a PDP-11/20 computer, which recorded all settings. The subjects pressed the center microswitch when a setting was complete, and this stored the response, erased the current display, and presented the next stimulus.
\end{abstract}

\section{Stimuli}

Sixteen different displays were derived from a basic Tolansky figure, shown in Figure 1b. The oblique parallels, when present, were $80 \mathrm{~mm}\left(4^{\circ}\right)$ long, separated by $15 \mathrm{~mm}$. All vertical lines were $10 \mathrm{~mm}$ long, and the oblique separation between the lower pair of lines (or dots) was $10 \mathrm{~mm}$. This differed from Day and Kasperczyk's 15-mm separation but was not considered an important parameter. Dots, in fact, were drawn as tiny "bow ties" (mirror-reflected triangles), but the triangles' sides were sufficiently small $(0.1 \mathrm{~mm}$, or $0.05^{\circ}$ ) to appear as a dot to the observer.

Of the 16 stimulus displays, two sets of 8 were identical, except that one set had oblique parallels present and the other set did not. Four of the 8 displays with parallels are shown in Figures $1 \mathrm{~b}, 1 \mathrm{c}$, $1 \mathrm{~d}$, and $1 \mathrm{e}$. The other 4 stimuli with parallels were inverted versions of these. For example, the inverted version of Figure $1 \mathrm{~b}$ had the same parallels arrangement, but the two vertical lines were on top and the single vertical was below. The subjects always adjusted the upper portion of the display. Thus, when the display was as in Figure $1 \mathrm{~b}$, the subjects caused the single upper line to slide along the upper parallel until it bisected the horizontal separation between the lower lines. When this display was inverted, the upper lines were moved until their virtual vertical bisector passed through the lower line.

One tap on a microswitch moved a stimulus line or dot $0.14 \mathrm{~mm}$. Continuous pressing produced apparently smooth movement, but, because the computer took longer to draw more complex stimuli (e.g., dots), the different stimulus arrangements moved at different speeds. These speeds were: one line, $0.90 \mathrm{~mm} / \mathrm{sec}$; one dot, $0.50 \mathrm{~mm} / \mathrm{sec}$; two lines, $0.44 \mathrm{~mm} / \mathrm{sec}$; and two dots, $0.25 \mathrm{~mm} / \mathrm{sec}$. However, these differences were not considered critical, because most of the subjects were conditioned by video games to tap; and even those who pressed continuously to begin with tended to tap slowly when close to a final setting.

To equate the luminance of all lines, the display luminance was set high but stimuli were viewed through partially crossed Polaroid filters surrounded by a black circular cardboard mask (when the luminance was not saturated in this way, line luminance varied with line orientation). The luminance of all lines (SEI photometer) was about $1.9 \mathrm{~cd} / \mathrm{m}^{2}$, and contrast, defined as $\left(L_{\max }-L_{\min }\right) /\left(L_{\max }+\right.$ $\left.L_{\min }\right)$ was close to 1.0 .

\section{Procedure}

Each subject made settings of all 16 displays, four times for each display, with starting positions of $\pm 2.5 \mathrm{~mm}$ and $\pm 5.0 \mathrm{~mm}$ relative to true bisection. These 64 trials were presented in a different random order to each subject. Each subject was dark adapted for a few minutes before the testing session began, and short rests were given when required, but always after the first half of the trials. 
In instructing subjects, we found that verbal instruction was not sufficient to eradicate confusion between vertical and oblique bisection. Therefore, the subjects were shown two drawings, one of which showed an upper dot vertically above the midpoint of two lower obliquely oriented dots, the other of which showed an upper dot lying on the virtual orthogonal bisector of the distance between the lower dots. On the first drawing, a vertical arrow pointed from the upper dot to the midpoint of the lower dots; on the second drawing, the arrow pointed obliquely from the upper dot to the midpoint between the lower pair. We stressed that the judgment required was the first one (vertical bisection) and that under no circumstances should oblique bisection be confused with it.

\section{Subjects}

There were 16 subjects, 9 volunteers from an introductory psychology course and 7 advanced undergraduate students. All were totally naive about the experiment, and all had emmetropic (normal) or corrected vision.

\section{RESULTS}

The mean settings for apparent bisection are shown in Figure 2. The left panel shows settings for the upright figures (as in Figure 1); the right panel shows settings for the inverted figures.

Labeling on the abscissa of Figure 2 indicates the nature of the stimulus display. For example, in the left-hand panel (upright figures) ${ }_{L L}^{L}$ indicates an upper vertical line and two lower vertical lines; $D$ indicates an upper dot and two lower vertical lines; $\mathrm{L}$ indicates an upper

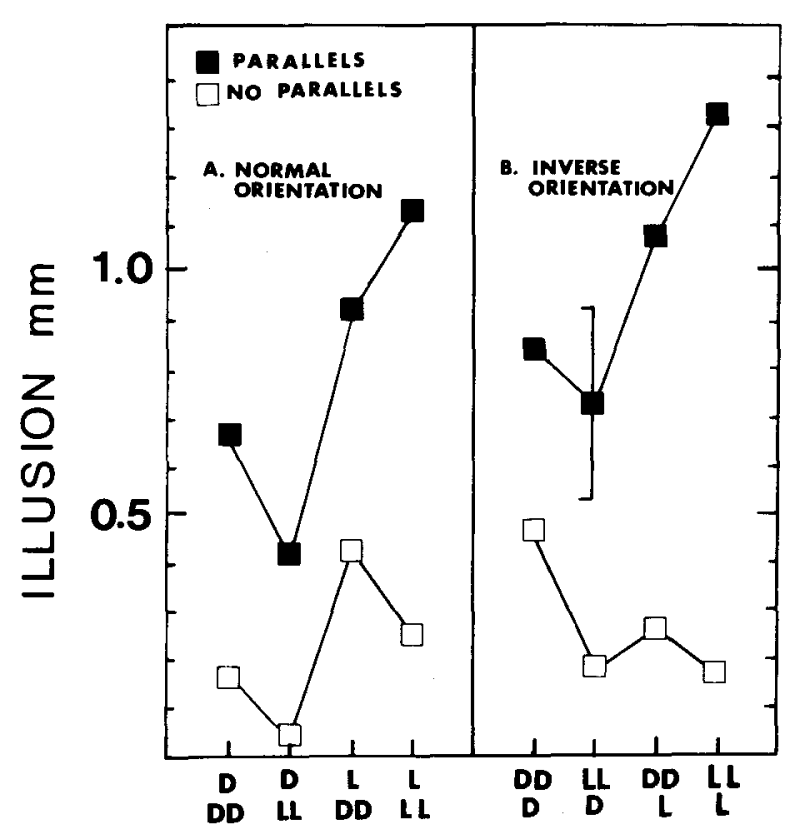

Figure 2. Mean illusions obtained with upright figures (left panel) and inverted figures (right panel) when oblique parallel lines are absent (open symbols) or present (filled symbols). Abscissa indicates nature of display, where $D=\operatorname{dot}(s), L=l i n e(s)$, and position of $D$ (or $L$ ), above or below, indicates whether the upper or lower transverse segment consisted of $\operatorname{dot}(\mathrm{s})$ or line(s). Thus, $\mathrm{LL}$ indicates a single upper dot and two lower lines. Positive illusions mean that the upper variable segment was set too low and to the right. Error bar in right panel shows \pm 1 standard error $\left(0.20^{\circ}\right)$. line and two lower dots; and $\underset{D D}{D}$ indicates an upper dot and two lower dots. For the inverted figures (right panel), the second mean from the left $\left(\begin{array}{c}\mathrm{DD} \\ \mathrm{L}\end{array}\right)$, for example, indicates two upper dots and a lower vertical line.

The open squares refer to figures without parallels, and the filled squares refer to figures with parallels. The data were analyzed using 15 planned orthogonal contrasts to discover which differences were significant, and we used a simple subjects $\times$ treatments design (Winer, 1962, chap. 4). In all cases, the critical value of $F(1,225)$ was 3.84 for $p=.05$.

The first eight contrasts compared the eight pairs of means in the left-hand and right-hand panels of Figure 2. Thus, each display with parallels (solid square) was contrasted with the same display without parallels (open square immediately below it). From left to right in Figure 2 these contrasts were: all dots, not significant $(F$ $=3.12)$, dot above, lines below, not significant $(F=$ $1.98)$, line above, dots below, not significant $(F=3.05)$, and line above, lines below, significant $(F=9.55$, $p<.005$ ). For the inverted figures, the corresponding contrasts gave $F=1.663, p>.05 ; F=3.60, p>.05 ; F$ $=8.23, p<.005$; and $F=16.60, p<.0005$. In sum, three of these eight contrasts were significant, and all involved cases in which the single pointer (whether above and moving as in the upright figures or below and stationary as in the inverted figures) was a line rather than a dot.

Four more contrasts averaged over means obtained with and without parallels and showed that it made no difference whether the pair of targets opposite the pointer was composed of dots or lines. The first of these contrasts compared upright dot-above means for figures with either dots below or lines below and was not significant $(F=$ 0.96). The second repeated this for the inverted figures and was also not significant $(F=0.97)$. The third compared upright line-above figures that had either lines or dots below; it was not significant $(F=0.005)$. The identical contrast for the inverted figures was also not significant $(F=0.15)$.

The overall mean of all upright figures with single upper lines differed from the overall mean of all figures with single upper dots $(F=6.47, p<.025)$, although the companion contrast for inverted figures was not significant $(F=1.25)$.

The overall mean for upright figures was not different from the overall mean for inverted figures $(F=1.77)$, although the obtained inverted means were bigger in six of the eight cases, including all of the four displays with parallels.

Finally, it was noted earlier that Day and Kasperczyk obtained a standard deviation three times larger in their three-dot condition than in their three-line condition (2.06 vs. 0.67 ). Under our instructions to avoid oblique bisection, this difference was reduced but not eradicated. The comparable standard deviations here were 1.47 and 0.83 . For the inverted figures, we obtained 1.28 and 0.96 ; for the parallel-less upright figures, 1.64 and 1.10; and for the parallel-less inverted figures, 1.38 and 1.13. This suggests that the extra instruction here may have reduced 
variability of settings for the three-dot display but that the three-dot task remains more difficult. However, Cochran's test of variance inhomogeneity (Winer, 1962) indicated that although the variances were significantly inhomogeneous in Day and Kasperczyk's experiment $[C(4,11)=0.34, p<.05]$, they were not so in the present case $[C(16,15)=0.14, p>.05]$.

\section{DISCUSSION}

The Poggendorff illusion historically has been attributed to distortions in the perceived size of the angles that occur at the intersect of the parallel inducing lines and the judged transversal (e.g., Helmholtz, 1910/1962; Hotopf \& Ollerearnshaw, 1972; Wenderoth, Beh, \& White, 1978). It has become clear from more recent research that, even if such angular effects do contribute to the illusion (and its variants), other factors must be involved. For example, manipulations of the adjustable portion of the transversal line, such as varying its orientation or replacing it with a dot, do not affect the illusion's magnitude in a manner consistent with a simple explanation couched in terms only of angular distortion (e.g., Day \& Dickinson, 1976; Tong \& Weintraub, 1974; Wenderoth, 1980, 1981; Wenderoth \& Johnson, 1981). Indeed, the fact that the illusion occurs, with reduced magnitude, in an all-dot display implicates factors other than angular and/or interactive line effects (Coren, 1970; Predebon, 1983; Wenderoth \& Wade, 1981).

There is, on the other hand, a great deal of evidence which shows that Poggendorff-type effects are larger when a transverse line intersects inducing parallels than when no parallels are present: the parallel-less Poggendorff illusion is quite small compared with the with-parallels effect (e.g., Curthoys, Wenderoth, \& Harris, 1975; Day, 1973; Goldstein \& Weintraub, 1972; Wundt, 1886).

The experiment reported here provides further evidence that the presence of inducing parallels increases the illusion when the transverse pointer is a line segment. Considering the four conditions in this study in which the adjustable pointer was a line (Figures $1 \mathrm{~b}, 1 \mathrm{~d}$, and their inverted versions), three of these four were found to increase significantly with the addition of the parallel lines. None of the effects in which the pointer was a dot (Figures 1c, 1e, and their inverted versions) increased with the addition of the parallels, although it can be noted that all obtained effects were larger when the parallels were present, suggesting a small (undetected) effect of adding parallels when the pointer is a dot. Also, for upright figures, the mean of all effects obtained with a line segment pointer was greater than the mean of all effects obtained with a dot pointer. A possible explanation is that when the pointer is a line, its orientation is distorted slightly (angle expansion) when it abuts a parallel and hence it must be displaced further down and to the right (in Figures $1 \mathrm{~b}$ and 1d) in order to appear to bisect the horizontal space between the lower targets. No such angular distortion would occur with a dot pointer. One pos- sibility worth considering, however, is that the addition of oblique parallels to the displays with dot pointers increases the salience of the oblique bisection alternative and biases subjects' settings in that direction.

Whether the lower pair of targets is composed of lines or dots should be of little (or lesser) consequence: these lines or dots merely define the extremities of the space to be bisected, and it should matter little whether these markers are seen as dots, truly vertical lines, or lines with an illusory tilt. This was borne out by the fact that no significant difference was found between figures with pairs of dot targets or pairs of line targets.

Originally, we had intended to run two experiments, one repeating the Day and Kasperczyk study without our instructions prohibiting oblique bisection and another with the special instructions. This would have shown objectively that the instruction is the variable that controls whether the three-dot effect with parallels does or does not differ from the three-line effect with parallels. However, as we described in the methods section, quite a number of our subjects showed confusion about the task in the absence of visual instruction (about $40 \%$ of subjects). Indeed, even with the graphic instruction we used, two subjects were still unable to comprehend the distinction between vertical and oblique bisection.

In addition, Day and Kasperczyk were not explicit regarding their own instructions. They stated that "subjects were instructed to move the upper element along the diagonal parallel so that it lay exactly halfway between the two lower ones, that is, so that if it were to be extended vertically downward it would exactly bisect the space between the two lower elements"' (Day \& Kasperczyk, 1985 , p. 76). It cannot be established from this report whether these were the exact words used or whether graphic displays, such as ours, were used to explicate the task. Hence, precise replication was not possible. If the instructions used were those quoted above, it might be the case that the words "lay exactly halfway between the two lower ones" were instrumental in causing some subjects to attempt oblique bisection or a compromise judgment.

This experiment has demonstrated, contrary to the assertion of Day and Kasperczyk, that displacement effects with dots on oblique parallels are not as great as those with lines on oblique parallels, when instructions are devised to eliminate subjects' confusion between vertical and oblique bisection, especially in the case of the all-dot stimulus. Accordingly, explanations of the Poggendorff illusion which include some contribution of line and angle interactions remain tenable. The fact that Day and Kasperczyk (1985) found no differences between the illusions induced by Figures $1 \mathrm{~b}$ to $1 \mathrm{e}$ could well be due partly to their subjects' tendency to compromise between vertical and oblique bisection in the three-dot task (Figure 1e), as they proposed, partly to the very small illusions which occur, and partly to the inherent difficulty of the tasks and the difficulty in conveying to the subjects the precise nature of the judgment to be made. 


\section{REFERENCES}

COREN, S. (1970). Lateral inhibition and geometric illusions. Quarterly Journal of Experimental Psychology, 22, 274-278.

Curthoys, I., Wenderoth, P., \& Harris, J. (1975). The effects of the motion path and the length of the variable segment in the Poggendorff illusion without parallels. Perception \& Psychophysics, 17, 358-362.

DAy, R. H. (1973). The oblique line illusion: The Poggendorff effect without parallels. Quarterly Journal of Experimental Psychology, 25, 535-541.

Day, R. H., \& Dickinson, R. G. (1976). The components of the Poggendorff illusion. British Journal of Psychology, 67, 537-552.

DAY, R. H., \& KASPERCZYK, R. T. (1985). Apparent displacement of lines and dots in a parallel-line figure: A clue to the basis of the Poggendorff effect. Perception \& Psychophysics, 38, 74-80.

Goldstein, M. B., \& WeinTraub, D. J. (1972). The parallel-less Poggendorff: Virtual contours put the illusion down but not out. Perception \& Psychophysics, 11, 353-355.

HeLmHOLTZ, H., vON (1962). Helmholtz's treatise on physiological optics (J. P. C. Southall, Trans.). New York: Dover. (Original work published 1910)

Hotopf, W. H. N., \& Ollerearnshaw, C. (1972). The regression to right angles tendency and the Poggendorff illusion. I. British Journal of Psychology, 63, 359-367.
Predebon, J. (1983). Recognition processes and the occurrence of the dot Poggendorff illusion. Perceptual \& Motor Skills, 36, 394.

Tong, L., \& Weintraub, D. J. (1974). Contour displacements and tracking errors: Probing 'twixt Poggendorff parallels. Perception \& Psychophysics, 15, 258-268.

WENDEROTH, P. (1980). Alignment errors in Poggendorff-like displays when the variable segment is a dot, a dot series, or a line. Perception \& Psychophysics, 27, 505-518.

Wenderoth, P. (1981). The role of the second oblique in the Poggendorff illusion. Perception, 10, 605-614.

Wenderoth, P., Beh, H., \& White, D. (1978). Perceptual distortion of an oblique line in the presence of an abutting vertical line. Vision Research, 18, 923-930.

Wenderoth, P., \& Johnson, M. (1981). Are the obtuse angles the key components of the Poggendorff illusion? Perception, 10, 165-172. Wenderoth, P., \& WADE, N. (1981). An investigation of line and dot forms of the Müller-Lyer and Poggendorff illusions. Quarterly Journal of Experimental Psychology, 33A, 77-85.

WINER, B. J. (1962). Statistical principles in experimental design. New York: McGraw-Hill.

WundT, W. (1886). Grundzüge der physiologischen Psychologie. Leipzig: Engelmann.

(Manuscript received December 1, 1985; revision accepted for publication March 26, 1986.) 